\title{
Editorial
}

\section{Sobre la eficacia de la formación medica continuada}

\author{
E. FERNÁNDEZ-MONDÉJAR
}

Hospital Universitario Virgen de las Nieves. Granada. España.

Los programas de formación médica continuada (FMC) constituyen un sistema eficaz para mejorar los conocimientos de los que los siguen, y asimismo pueden ser útiles a la hora de cumplir determinados objetivos relacionados con la carrera profesional. Sin embargo, el planteamiento deberá ser más ambicioso y, además de lo anterior, la FMC debería ser capaz de mejorar nuestra actividad clínica diaria, y esto debería redundar en un mejor pronóstico de los pacientes. Así como los primeros dos puntos sí parecen fácilmente asequibles, es muy dudoso que se alcance los últimos dos con los diferentes programas de $\mathrm{FMC}^{1}$, o al menos los resultados son tan difusos y dalatados en el tiempo que es difícil establecer una relacion causa efecto.

Después de 1 año desde la puesta en marcha del programa de FMC de nuestra revista, conviene analizar la situación y plantear las necesarias modificaciones tendentes a conseguir una mayor eficacia.

Un planteamiento lógico para la implantación de programas de FMC debería consistir en: a) identificar áreas clínicas mejorables; b) realizar acciones de FMC tendentes a cubrir esos déficit, y c) evaluar su eficacia no sólo en cuanto a la mejora de conocimientos, sino como impacto en salud.

¿Quíen debe detectar las áreas clínicas mejorables? Por supuesto, cada médico ha de tener la suficiente capacidad de autocrítica para identificar sus limitaciones y plantear su solución mediante la oferta de FMC. Éste es uno de los fundamentos básicos para el Desarrollo Profesional Continuo ${ }^{2}$. No obstante, hemos de reconocer que en determinadas circunstancias la función del médico se encuentra inmersa en un sistema de salud que responde a intereses generales, donde se mezclan necesidades

Correspondencia: Dr. E. Fernández Mondéjar.

Hospital Universitario Virgen de las Nieves.

Ctra. de Jaén, s/n. 18013 Granada. España.

Correo electrónico: enrique.fernandez.mondejar.sspa@juntadeandalucia.es no puramente sanitarias, sino sociales, y en el que el médico tiene una función técnica muy precisa. En este sentido, los sistemas de salud y otras entidades como las sociedades científicas tienen el derecho e incluso la obligación de detectar las áreas deficitarias en las que sus médicos o afiliados deben formarse.

Desde Medicina Intensiva hemos planteado un sistema de FMC basado en preguntas sobre los artículos publicados (originales y revisiones). En un plano realista, el sistema está orientado a mejorar los conocimientos de los lectores y a satisfacer las necesidades de acreditación exigidas para la carrera profesional. Por supuesto que no renunciamos a otros propósitos más ambiciosos, como la mejora en la práctica clínica diaria y su repercusión en el pronóstico, aunque la evaluación de estos extremos, como ya se ha comentado, es enormemente dificil y probablemente no depende de una actividad concreta, sino de un conjunto de acciones formativas que favorezcan un cambio de actitud.

Hasta ahora, con nuestro sistema de FMC se podían obtener 12,2 créditos al año ( 9 números de la revista) con un planteamiento de «todo o nada». Sin duda este número de créditos supone un atractivo importante, pero la contrapartida es la rigidez del programa, ya que requiere su continuidad durante 1 año. A pesar de estas limitaciones, durante este primer año, 261 médicos han completado nuestro programa de FMC. No obstante, muchos intensivistas no pueden comprometerse a un seguimiento mantenido durante un periodo tan largo y podrían acceder a nuestro programa de forma parcial o intermitente, aunque obtengan un número de créditos menor. Por este motivo, en las próximas ediciones el formato será diferente, y se asignará 2,1 créditos por cada número de la revista respondido adecuadamente. De esta forma, cada lector podrá decidir cuántos créditos desea obtener. También se gana en flexibilidad, ya que se podrá decidir a qué números de la revista desea acceder, 
con lo que podría cubrir las carencias formativas que haya identificado.

Esperamos que estos cambios en nuestro programa de FMC mejoren su eficacia y sean de utilidad para la formación de nuestros lectores, e idealmente esto redunde en una mejora en su actividad clínica diaria.

\section{BIBLIOGRAFÍA}

1. Boonyasai RT, Windish DM, Chakraborti C, Feldman LS, Rubin HR, Bass EB. Effectivness of teaching quality improvement to clinicians: a systematic review. JAMA. 2007;298:1023-37.

2. Fernández Mondejar E, Alía I. Formación médica continuada. Una obligación compartida. Med Intensiva. 2008;32:1-2. 